\title{
Pictures, words, and the structure of the trace in immediate recall
}

\author{
MICHAEL C. KING and WILLIAM BEVAN \\ Duke University, Durham, North Carolina 27706
}

\begin{abstract}
The reduction-cuing method was used to investigate the trace structure associated with both pictorial and verbal stimuli. The results indicate that those aspects of the trace assumed to be encoded as both pictorial and verbal mediators were in fact accessible to pictorial cues, but not to word cues. Furthermore, no evidence was obtained for any aspect of the trace composed of verbal but not pictorial elements. These outcomes are not consistent with a strict dual coding hypothesis, but they can be interpreted within the context of a transformational hypothesis.
\end{abstract}

Questions concerning the nature of the stimulus trace continue to challenge the curiosity of experimental psychologists. In recent years, one dominant focus of interest has centered about issues relating to the relative roles of pictorial and linguistic processes in mediating recall. The present investigation seeks to approach this problem through the strategy of the reduction-cuing method (Tulving \& Bower, 1974; Tulving \& Watkins, 1975).

The reduction-cuing method provides an economical means of describing trace structure. Its logic may be briefly summarized as follows (see Tulving \& Watkins, 1975, for a fuller description): The observer is presented a set of stimuli for later recall. Subsequently, two fragments of each stimulus (Cue X and Cue Y) are sequentially presented as aids to recall. With half the stimuli, Cue X precedes Cue Y; with the remaining half, Cue $Y$ precedes Cue $X$. When the test trials have been completed, the frequency of recall of each stimulus in the presence of each cue is entered into one or the other of two 2 by 2 contingency tables, one for each temporal order in which the cues were presented. The reduction method transforms these two data matrices into a trace matrix that is assumed to describe the structure of the memory trace in terms of cue components independent of cuing sequence.

If one takes $R$ to represent recall, $\bar{R}$ to represent failure of recall, and the numerals 1 and 2 to represent Cue $X$ and Cue $Y$, respectively, then the $R_{1} R_{2}$ entry in the trace matrix reflects recall attributable to both cues. This is called the common valence and is assumed to represent those aspects of the trace composed of processes associated with both cues. By the same token, $\overline{R_{1}} R_{2}$ and $R_{1} \overline{R_{2}}$, called the reduced valences, are assumed to represent those aspects of the trace composed of processes associated with one but not the other cue. Similarly, $\overline{R_{1} R_{2}}$ is assumed to represent aspects of the trace associated with neither of the stimulus cues. Finally, the $R_{1}$ and $R_{2}$ sums represent the proportion of recall associated with each cue and are known as gross valences.
In the present experiment, observers were presented a series of labeled drawings as stimuli for recall. Each drawing depicted a simple action involving an agent and an object acted upon. The label consisted of a written trigram (noun-verb-noun) describing the drawing. Recall of each stimulus was tested twice in the presence of 1 of 16 cue-pair conditions. Each cuing condition was formed by the exhaustive combination of individually presented single or double cues that were either pictorial (the drawing of the agent or drawings of both agent and object) or verbal (the subject noun or both subject and object nouns). Recall in all trials was with the trigram verb describing the action.

According to Paivio's $(1969,1975)$ dual coding hypothesis of recall, separate, functionally independent memory codes exist for pictorial and for verbal stimuli. If such is indeed the case, then the ability of the trace associated with a particular kind of cue to mediate recall (the valence obtained for the cue by means of the reduction method) should be at least partially independent of that associated with a cue for the same event from the alternate modality. Within the paradigm of the reduction method, this independence would be indicated by reduced valences in the trace matrix that are greater than zero for mixed-cue pairs, and complete independence would be indicated by both reduced valences being greater than zero. The present experiment tests the prediction that reduced valences for mixed pictureword cue pairs should be greater than zero for both pictorial and verbal cues. A particularly strong test occurs when single cues of one type (either drawing or word) are paired with double cues of the alternative type.

\section{METHOD}

\begin{abstract}
Design
Sixteen pairs of cues were formed by making all possible combinations of pairs of the four cues (one drawing, two drawings, one word, two words). Cue pairs were then counterbalanced across both stimuli and observers. Each pair was matched twice across the 16 observers with each of 32 stimuli by means of two
\end{abstract}


Latin squares. The position of each stimulus in the presentation sequence was counterbalanced within both odd and even positions. Thus each of Stimuli 1-16 occurred equally often across observers in the even positions, and each of Stimuli 17-32 occurred equally often in the odd positions. This precaution was taken to minimize the possibility of serial position effects when recall data were averaged. The position of each cue in the recall sequence of 64 trials for individual observers was determined by pairs of random numbers.

\section{Subjects}

Sixteen Duke University undergraduates (12 female and 4 male) served as observers.

\section{Materials \\ The 32 triplets that served as verbal stimuli (see Table 1) were typed on white paper. The stimulus drawings with which they were matched were executed in India ink on white paper by a local artist. Drawings and attached typed labels were then photographed and made into slides to be used as initial stimuli. In addition, four cue slides were prepared as follows for use with each initial stimulus: (1) a drawing of the agent depicted in the drawing (single-pictorial, or 1P, cue), (2) a drawing of both agent and object (double-pictorial, or $2 \mathrm{P}$, cue), (3) the generic name of the agent (single-word, or $1 \mathrm{~W}$, cue), and (4) the names of the agent and the object (double-word, or $2 \mathrm{~W}$, cue). Drawings used as cues were exact copies of the relevant portions of the original stimulus drawings, with agent and object separated such that no action between the two was implied. Care was taken in constructing the triplets such that the interaction between agent and object depicted in the drawing was not automatically implied by the identity of either.}

\section{Procedure}

Individual observers were seated at a table approximately $5 \mathrm{ft}$ from the screen on which stimuli were to be presented. Each was informed that he or she would be shown two sequences of slides, the first sequence to consist of 32 slides depicting events to be recalled, the second to consist of 64 slides that would contain cues to aid in recall. The concept of the triplet was next explained, as was that of single and double cues. Observers were then instructed to respond on each cued recall trial by writing down the verb for the stimulus triplet on a specially prepared answer sheet. They were specifically admonished not to look at past answers, but to feel free to guess when they were not certain of the identity of the verb. A Kodak Carousel projector was used to present slides for both sequences at a rate of approximately $7 \mathrm{sec}$ per slide and with an

Table 1

The 32 Triplets Constructed for the Present Study

\begin{tabular}{ll} 
1. cowboy ride horse & 17. letter cue butter \\
2. tank ram tomb & 18. ambulance hit building \\
3. elephant chase beaver & 19. bottle contain pencil \\
4. skillet fry goblet & 20. mule kick cabin \\
5. table support clock & 21. truck haul yacht \\
6. microscope examine dollar & 22. missile orbit earth \\
7. tweezers grip coin & 23. star illuminate palace \\
8. cane balance plant & 24. revolver shoot apple \\
9. fork spear lobster & 25. prisoner carry board \\
10. cradle rock frog & 26. tree shade house \\
11. boy drag doll & 27. ink stain dress \\
12. doctor hold umbrella & 28. noose hang corn \\
13. oven bake trumpet & 29. fire destroy church \\
14. refrigerator chill book & 30 . hammer break window \\
15. scorpion lift tablespoon & 31. robber jump hurdle \\
16. caterpillar cross magazine & 32 . mosquito bite cigar \\
\hline
\end{tabular}

interval of approximately $1 \mathrm{sec}$ between slides. The cued recall sequence followed the initial stimulus sequence as rapidly as the experimenter could change slide trays. The entire experimental session, including debriefing, took less than 30 min per observer.

\section{RESULTS}

Responses were scored correct if the observer responded with the verb from the appropriate triplet. Variations in tense were accepted as correct, but synonyms were not. Recall data were entered into 16 2 by 2 contingency matrices, 1 for each cue-pair condition. These data represented the responses on a total of 1,024 trials. Errors occurred on 275 trials, yielding an overall error rate of $27 \%$ (mean errors per observer = $17.2 ; \mathrm{SD}=8.2$ ). Types of errors were distributed as follows: first-cue errors, 147; second-cue errors, 128; errors with pictorial cues, 127; errors with verbal cues, 148.

One of the assumptions of the reduction method is that the no-recall cells $\left(\overline{R_{1} R_{2}}\right)$ in the two data matrices used to construct a trace matrix should be equal(Tulving \& Watkins, 1975). This assumption was not met in the present experiment, since our $\overline{R_{1} R_{2}}$ cells differed by as much as .28. The implications of this violation are at present not clear.

Table 2 presents valences for the several combinations of sequential cues. The rows of this table represent different ways of averaging reduction-cue data. For example, Row 1 reports average valences derived from all the 2 by 2 data matrices involving single and double cues. Row 12 contains valences derived from the two data matrices resulting from the $1 \mathrm{P}-1 \mathrm{~W}$ condition.

Inspection of the reduced valences in Rows 1 and 2 indicates that when traces were reduced either by word cues or by single cues, a substantial portion of the trace was still accessible to pictorial or double cues (.09 and .07 , respectively).

Rows 3-6 present valences for those conditions when the sequenced cues were of the same type. An examination of these cues provides information concerning whether the repetition of cues facilitates recall. Except where verbal cues were repeated, the answer is negative, since all reduced valences except for the repetition of $1 \mathrm{~W}$ and $2 \mathrm{~W}$ cues are zero.

Rows 7-10 involve pitting each cue type against the average of all others. It will be noted that all reduced valences for the $R_{1} \overline{R_{2}}$ condition are approximately equal. Meanwhile, the reduced valences for the two conditions involving verbal cues are clearly larger than those involving pictorial cues. This suggests that the trace is accessible to other cues after reduction by verbal cues, but not after pictorial cues.

Finally, Rows 11-16 include valences for each of the several mixed-cue conditions. Inspection indicates that the gross valence associated with a $1 \mathrm{~W}$ cue, $R_{1}$ or $R_{2}$, is low for every pair. In addition, large reduced valences 
Table 2

Trace Matrices as Balanced by the Reduction Method

\begin{tabular}{|c|c|c|c|c|c|c|c|c|c|}
\hline \multirow[b]{2}{*}{ Row } & \multirow[b]{2}{*}{ Cue Condition } & \multicolumn{8}{|c|}{ Recall Valences } \\
\hline & & $\mathrm{R}_{1}$ & $\overline{R_{1}}$ & $R_{1} R_{2}$ & $R_{1} \overline{R_{2}}$ & $R_{1} \overline{R_{2}}$ & $\overline{R_{1} R_{2}}$ & $\mathbf{R}_{2}$ & $\overline{R_{2}}$ \\
\hline 1 & Single-Double & .69 & .31 & .66 & .07 & .03 & .24 & .73 & .27 \\
\hline 2 & Picture-Word & .76 & .24 & .67 & .03 & .09 & .22 & .69 & .31 \\
\hline 3 & 1P-1P & .72 & .28 & .72 & 0 & 0 & .28 & .72 & .28 \\
\hline 4 & $2 \mathrm{P}-2 \mathrm{P}$ & .75 & .25 & .75 & 0 & 0 & .25 & .75 & .25 \\
\hline 5 & $1 \mathrm{~W}-1 \mathrm{~W}$ & .59 & .41 & .59 & .13 & 0 & .28 & .72 & .28 \\
\hline 6 & $2 W-2 W$ & .75 & .25 & .75 & .03 & 0 & .22 & .78 & .22 \\
\hline 7 & 1P-Overall & .73 & .27 & .69 & .02 & .04 & .25 & .71 & .29 \\
\hline 8 & 2P-Overall & .78 & .22 & .74 & 0 & .04 & .22 & .74 & .26 \\
\hline 9 & 1W-Overall & .60 & .40 & .55 & .12 & .05 & .28 & .67 & .33 \\
\hline 10 & 2W-Overall & .72 & .28 & .67 & .06 & .05 & .22 & .73 & .27 \\
\hline 11 & $1 \mathrm{P}-2 \mathrm{P}$ & .73 & .27 & .73 & .03 & 0 & .24 & .76 & .2 \\
\hline 12 & $1 \mathrm{P}-1 \mathrm{~W}$ & .70 & .30 & .57 & 0 & .13 & .30 & .57 & .43 \\
\hline 13 & $1 \mathrm{P}-2 \mathrm{~W}$ & .76 & .24 & .73 & .06 & .03 & .18 & .79 & .21 \\
\hline 14 & $2 \mathrm{P}-1 \mathrm{~W}$ & .76 & .24 & .67 & 0 & .09 & .24 & .67 & .33 \\
\hline 15 & $2 \mathrm{P}-2 \mathrm{~W}$ & .82 & .18 & .76 & 0 & .06 & .18 & .76 & .24 \\
\hline 16 & $1 \mathrm{~W}-2 \mathrm{~W}$ & .59 & .41 & .52 & .11 & .07 & .30 & .63 & .37 \\
\hline
\end{tabular}

Note-Rows indicate different trace matrices (2 by 2 contingency tables). Columns indicate entries in each table formed by the balanced probability of recall $(R)$ and no recall $(R)$ of the leftmost cue (1) and rightmost cue (2) in column 2 . (See text for full description.)

are found in connection with the $1 \mathrm{P}$ condition in Row 12 , the $2 \mathrm{P}$ condition in Row 14 , and the $2 \mathrm{~W}$ condition in Row 16 . Rows 12,14 , and 15 reveal the reduced valence for the trace component $\overline{R_{1}} R_{2}$ to be zero when the valence for $R_{1} \overline{R_{2}}$ is large. This suggests that cuing with pictures in mixed pairs results in access to parts of the trace not available to verbal cues but not vice versa.

When the paired cues are of the same type (Rows 11 and 16), the pattern of reduced valences is different. Almost all of the traces associated with $2 \mathrm{P}$ cues are accessible to $1 \mathrm{P}$ cues. Essentially, the same thing can be said of the results with $1 \mathrm{~W}$ and $2 \mathrm{~W}$ cues. Meanwhile, the large reduced valences in the $1 \mathrm{~W}-2 \mathrm{~W}$ combination imply that memory traces of the sort generated in the context of this experiment were largely composed of processes other than those associated with either verbal cue. This finding is rather unexpected when considered in the context of the dual coding hypothesis.

\section{DISCUSSION}

The data of the present experiment, taken together, do not support the dual coding hypothesis when strictly defined, for they indicate that, while there are aspects of the memory trace accessible to pictorial cues that are at the same time immune to verbal cues, no part of the trace is simultaneously accessible to verbal cues and inaccessible to pictorial cues.
This asymmetrical outcome can be predicted from a transformational hypothesis. If we assume that, upon seeing a pictorial cue, the observer names the picture and subsequently uses both pictorial and verbal aspects of the trace process in mediation, then we should find high overall recall with pictorial cues as well as large reduced valences associated with the pictorial components of mixed cues. This is what is seen in the present data. If, however, the observer attempts to call up a pictorial process in the presence of a verbal cue, he or she is faced with a far more difficult task. Thus it may be expected that he or she will less frequently produce the correct combination of verbal and pictorial mediators than was true in the first instance, and recall in the presence of verbal cues will generally be poorer. In addition, the repetition of verbal cues should increase the probability of making contact with the correct pictorial process and thereby produce a high reduced valence for a second word cue, a result also found in the present data.

\section{REFERENCES}

Paivio, A. Mental imagery in associative learning and memory. Psychological Review, 1969, 76, 241-263.

Paivio, A. Coding distinctions and repetition effects in memory. In G. Bower (Ed.), The psychology of learning and motivation (Vol. 9). New York: Academic Press, 1975.

Tulving, E., \& Bower, G. H. The logic of memory representations. In G. Bower (Ed.), The psychology of learning and motivation (Vol. 8). New York: Academic Press, 1974.

Tulving, E., \& Watkins, M. J. Structure of memory traces. Psychological Review, 1975, 82, 261-275.

(Received for publication May 23, 1979.) 